\title{
ARTICLE
}

\section{AMPK maintains energy homeostasis and survival in cancer cells via regulating $\mathrm{p} 38 / \mathrm{PGC}-1 \alpha$-mediated mitochondrial biogenesis}

\author{
B Chaube ${ }^{1}, \mathrm{P}$ Malvi ${ }^{1}$, SV Singh ${ }^{1}$, N Mohammad ${ }^{1}$, B Viollet ${ }^{2,3,4}$ and MK Bhat ${ }^{1}$
}

\begin{abstract}
Cancer cells exhibit unique metabolic response and adaptation to the fluctuating microenvironment, yet molecular and biochemical events imprinting this phenomenon are unclear. Here, we show that metabolic homeostasis and adaptation to metabolic stress in cancer cells are primarily achieved by an integrated response exerted by the activation of AMPK. We provide evidence that AMPK-p38-PGC-1a axis, by regulating energy homeostasis, maintains survival in cancer cells under glucose-limiting conditions. Functioning as a molecular switch, AMPK promotes glycolysis by activating PFK2, and facilitates mitochondrial metabolism of non-glucose carbon sources thereby maintaining cellular ATP level. Interestingly, we noted that AMPK can promote oxidative metabolism via increasing mitochondrial biogenesis and OXPHOS capacity via regulating expression of PGC-1a through p38MAPK activation. Taken together, our study signifies the fundamental role of AMPK in controlling cellular bioenergetics and mitochondrial biogenesis in cancer cells.
\end{abstract}

Cell Death Discovery (2015) 1, 15063; doi:10.1038/cddiscovery.2015.63; published online 21 December 2015

\section{INTRODUCTION}

Altered glucose metabolism is a characteristic feature of rapidly growing cancer cells. ${ }^{1,2}$ Cancer cells metabolize glucose primarily through aerobic glycolysis. ${ }^{2}$ Enhanced glycolysis is considered as one of the hallmarks of aggressive tumors. Nonetheless, recently, it has been shown that functional mitochondria are vital for tumorigenesis. ${ }^{3-6}$ Unlike normal tissues, tumors have more dense structure and irregular distribution of blood vessels owing to the immense ability of cancer cells to proliferate. In solid tumors, various stress conditions like low nutrient availability, energy depletion, hypoxia and oxidative stress arise during excessive growth and proliferation. ${ }^{7}$ Owing to the heterogeneous distribution of oxygen, glucose, glutamine and other nutrients in the solid tumor, cells have to adapt to nutritionally stressed microenvironment which confers selective survival advantage. A question still remains to be answered as to how cancer cells cope up with these tribulations to achieve survival, and simultaneously maintain rapid growth and proliferation. Given the heterogeneous nature of tumor microenvironment, there must be adaptive mechanisms that can maintain energy and metabolic homeostasis. Unfortunately, the nature of actual metabolic remodeling in cancer cells has often been veiled owing to the use of in vitro cell culture condition that provides high glucose and oxygen in contrary to the actual situation found in tumor microenvironment. It is well known that chronic energy deprivation and metabolic stress results in elevated mitochondrial oxidative capacity in muscles cells by inducing mitochondrial biogenesis. ${ }^{8-10}$ However, in cancer cells, despite high levels of physiological stress, the role of mitochondria in maintaining cell survival and homeostasis is not very clear.
All the cells have specific energy and nutrient sensors like AMP-activated protein kinase (AMPK) and mammalian target of rapamycin (mTOR). AMPK, upon energy depletion, initiates signaling cascade resulting in the suppression of ATP consuming pathways with concomitant induction of biochemical reactions that generate ATP. ${ }^{11}$ AMPK serves as a fuel gauge as it is activated by low ATP/AMP ratio, and is thought to protect mammalian cells against energy deprivation by controlling various pathways to maintain energy homeostasis. ${ }^{12}$ Conversely, mTOR is a master regulator of cell growth and proliferation under nutrientabundant conditions. ${ }^{13}$ AMPK is known to inhibit mTOR by directly phosphorylating raptor, one of the molecules of TOR complex. ${ }^{11,13}$ Most of the reports suggest that AMPK is a tumor suppressor as it inhibits many pathways involved in growth and proliferation. ${ }^{11}$ Other than regulating metabolism, it is also believed to regulate expression of genes associated with metabolism via localizing to the nuclei of many cells. ${ }^{14,15}$ Recent correlative studies suggest that AMPK increases mitochondrial biogenesis ${ }^{8}$ and OXPHOS capacity $^{16}$ in rat skeletal muscles. It has been shown that peroxisome proliferator-activated receptor coactivator-1 (PGC-1a) is involved in mitochondrial biogenesis in muscles. ${ }^{17,18}$ However, the mechanisms controlling mitochondrial biogenesis and cell survival under metabolic stress in cancer cells are not very clear. To examine whether AMPK is involved in mitochondrial biogenesis so as to provide survival advantage to cancer cells under glucose-limiting conditions, we used both biochemical as well as genetic approaches.

Herein, we describe that activation of AMPK is required for increased mitochondrial biogenesis in response to glucose limitation. Furthermore, we show that AMPK activation is required for increased expression of PGC-1a and TFAM. Expression of

\footnotetext{
${ }^{1}$ National Centre for Cell Science, Savitribai Phule Pune University Campus, Ganeshkhind, Pune, Maharashtra 411007 , India; ${ }^{2}$ INSERM, U1016, Institut Cochin, Paris, France; ${ }^{3}$ CNRS, UMR 8104, Paris, France and ${ }^{4}$ Université Paris Descartes, Sorbonne Paris Cité, Paris, France.

Correspondence: MK Bhat (manojkbhat@nccs.res.in or manojkbhat62@gmail.com)

Received 1 October 2015; revised 5 November 2015; accepted 6 November 2015; Edited by R Aqeilan
} 
PGC-1a is controlled by AMPK-induced activation of p38MAPK. Overall, this study highlights the role of AMPK in controlling cellular bioenergetics and mitochondrial biogenesis in cancer cells under glucose-limiting conditions.

\section{RESULTS}

AMPK protects cancer cells from glucose deprivation-induced death

Considering the heterogeneity and physiological stress in tumor microenvironment, we hypothesized that under metabolic stress, cells survive by activating AMPK to maintain energy and metabolic homeostasis. To investigate the involvement of AMPK in cell survival, we used $\mathrm{H} 1299$ cells stably transfected with dominant negative form of AMPK-a1 subunit (H1299-DN). We observed that H1299-DN cells failed to survive under glucose-limiting conditions despite the activation of AMPK by AICAR as compared with $\mathrm{H} 1299-\mathrm{EV}$ cells (Figures $1 \mathrm{a}$ and b). This finding was consistent with siRNA-mediated knockdown of AMPK-a1/2 in H1299 cells (Figure 1c). Further, to confirm the cell-protecting role of AMPK we used transformed AMPK-a1/a2 double knockout (AMPK-DKO) MEFs. In parallel, matching wild-type MEF (WT-MEF) were used as control. WT-MEF treated with AICAR survived under nutritional stress, whereas DKO cells failed to survive even upon AICAR treatment (Figures 1d and e, and Supplementary Figures S1a-e). a

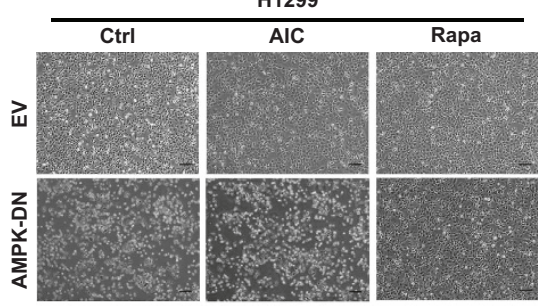

e
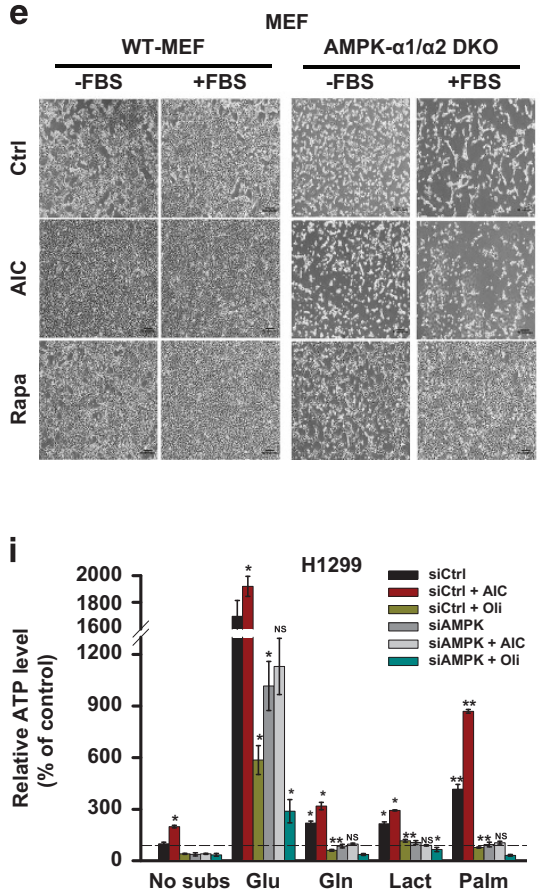

b

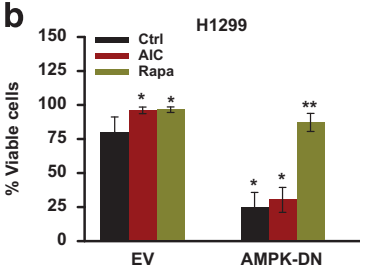

C

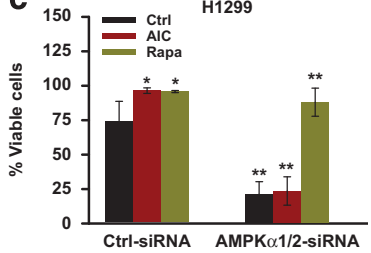

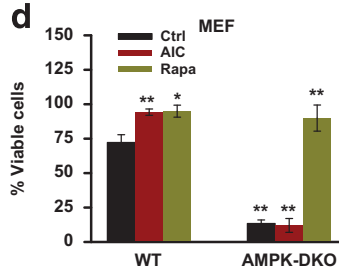

g f
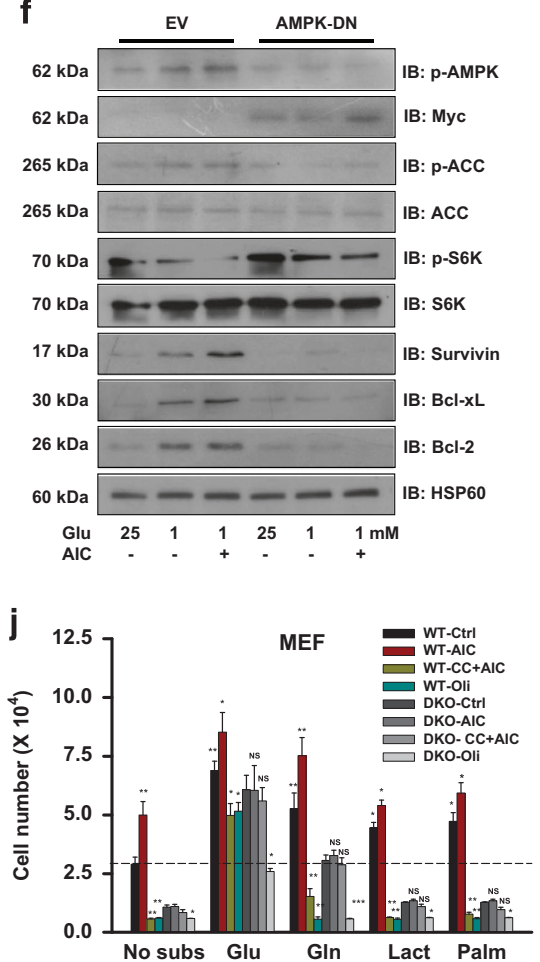
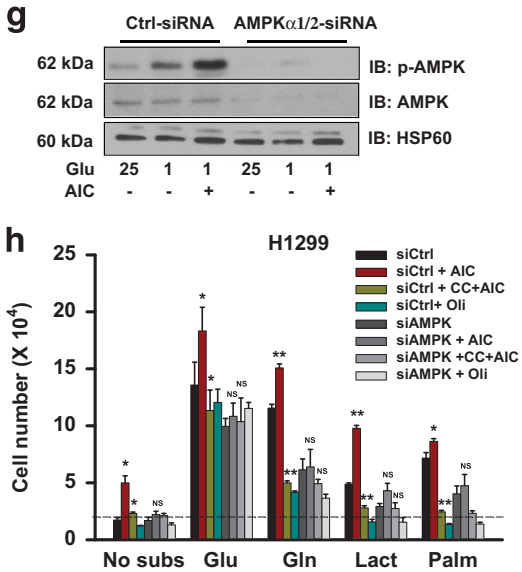

k

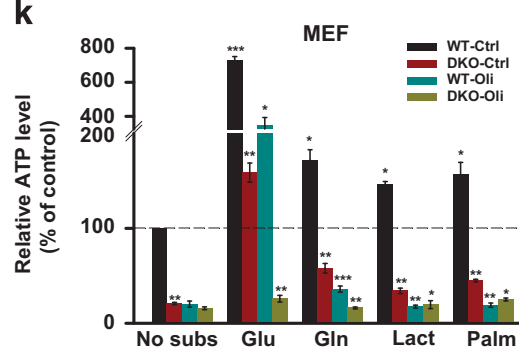

Figure 1. AMPK maintains cell survival under metabolic stress by promoting metabolism of non-glucose carbon sources. (a) Images representing the cellular morphology of $\mathrm{H} 1299$ cells stably transfected with either pCDNA3 vector (H1299-EV) or with dominant-negative form of AMPK-a1 subunits tagged with Myc (H1299-DN). These cells were grown under glucose-limiting conditions (1 mM glucose, $2 \mathrm{mM}$ glutamine and $10 \% \mathrm{FBS}$ ) in the presence or absence of $0.5 \mathrm{mM}$ AICAR (AIC) or $20 \mathrm{nM}$ rapamycin (Rapa) for $36 \mathrm{~h}$. (Scale bar, $50 \mu \mathrm{m}$ ). (b) Viability of H1299-EV and H1299-DN cells cultured under the conditions mentioned in a for $36 \mathrm{~h}$, as assessed by PI staining. (c) H1299 cells were transfected with AMPK-a1/2-specific siRNA and grown under conditions used in b, viability of these cells was assessed by PI staining. (d) Viability of AMPK- $a 1 / 2^{+/+}$(WT) and AMPK- $a 1 / 2^{-1-}$ (DKO) MEFs cultured under the conditions mentioned in a for $36 \mathrm{~h}$, as assessed by PI staining. (e) Representative images showing cellular morphology of WT and AMPK-DKO MEFs cultured in DMEM with or without glucose and FBS in the presence or absence of $0.5 \mathrm{mM}$ AICAR or $20 \mathrm{nM}$ rapamycin for $36 \mathrm{~h}$ (Scale bar, $50 \mu \mathrm{m}$ ). (f) H1299-EV and H1299-DN cells were grown in either 25 or $1 \mathrm{mM}$ glucose in the presence or absence of $0.5 \mathrm{mM}$ AICAR for $24 \mathrm{~h}$. Representative immunoblots showing the levels of indicated molecules. Antibody for Myc tag (9E10) was used for detecting overexpressed dominant-negative form of AMPK a1 subunit. HSP60 was used as a loading control. (g) Representative immunoblots showing phosphorylated and basal levels of AMPK in H1299 cells transfected with either control or AMPK-a1/2-specific siRNAs. HSP60 was used as loading control. (h) Survival and (i) ATP level in H1299 cells (transfected with AMPK- $a 1 / 2$ siRNA) cultured in glucose and glutamine-free DMEM supplemented with $5 \mathrm{mM}$ glucose (Glu), $5 \mathrm{mM}$ glutamine (Gln), $5 \mathrm{mM}$ galactose (Gal), $10 \mathrm{mM}$ lactate (Lact) and $100 \mu \mathrm{M}$ palmitate (Palm) in the presence or absence of $0.5 \mathrm{mM}$ AICAR (AIC), $10 \mu \mathrm{M} \mathrm{compound} \mathrm{C} \mathrm{(CC)}$ and $1 \mu \mathrm{M}$ oligomycin (Oli) for $48 \mathrm{~h}$. (j, k) Survival and relative ATP level in WT and DKO MEFs cultured under similar conditions used in $\mathbf{h}$ and $\mathbf{i}$. Values are represented as mean \pm S.D. ${ }^{*} P<0.05,{ }^{*} P<0.01,{ }^{* *} P<0.001$ denote significant differences between the groups. 
As AMPK is known to suppress mTOR that is involved in cell proliferation, we hypothesized that inhibition of mTOR might support cell survival under glucose-limiting conditions. Irrespective of AMPK status, rapamycin (an inhibitor of mTOR) promoted survival in H1299 as well as MEF cells under metabolic stress (Figures 1a-e).

Further, we checked molecular events involved in the survival of H1299-EV or H1299-DN cells grown in $1 \mathrm{mM}$ glucose in the presence or absence of AICAR. We found that glucose-limiting condition activated AMPK, which was further augmented upon treatment with AICAR in H1299-EV cells in comparison with H1299-DN cells as reflected by phosphorylated levels of ACC (Figure 1f). Using AMPK-a1/2-specific siRNA, we observed similar trend in the activation of AMPK by AICAR and/or glucose-limiting conditions (Figure 1g). Moreover, the activation of AMPK resulted in suppression of mTOR pathway as evident by decreased phosphorylation of p70-S6Kinase (Figure 1f). Next, we checked the levels of molecules associated with the survival of H1299-EV and H1299-DN cells grown under glucose-limiting conditions. Increase in protein levels of survivin, $\mathrm{BCl}-\mathrm{xL}$ and $\mathrm{BCl}-2$ was detected in H1299-EV cells under glucose-limiting conditions, which was further elevated upon AICAR treatment, whereas no change in these molecules was observed in H1299-DN cells under similar conditions (Figure 1f). These results indicate that AMPK-mediated cell survival is dependent on inhibition of mTOR.

\section{AMPK facilitates mitochondrial metabolism of non-glucose} substrates under metabolic stress

Balance between energy generation and utilization dictates cell survival under limiting nutrient availability or metabolic stress. ${ }^{19}$ When glucose availability is limited or diminished, cancer cell utilizes non-glucose carbon sources like glutamine, lactate and fatty acids. We asked whether AMPK is involved in facilitating metabolism of these substrates under glucose-deprived conditions. Noticeably, AMPK knocked-down H1299 cells failed to grow in the presence of glutamine, lactate and palmitate in the absence of glucose as compared with control cells (Figure $1 \mathrm{~h}$ ). Treatment with AICAR increased survival, which was reversed by the addition of compound $\mathrm{C}$ in control H1299 cells as compared with siAMPK cells (Figure $1 \mathrm{~h}$ ). With the presumption that maintenance of energy balance is the first step associated with AMPK-mediated cell survival, we measured ATP level under varying nutritional status. We noticed increased ATP level in cancer cells upon AMPK activation. Under identical conditions, H1299 (siCtrl) cells treated with AICAR generated more ATP in the presence of glutamine, lactate and palmitate, which is reflected by prolonged survival as compared with cells upon AMPK knockdown (Figures $1 \mathrm{~h}$ and i; Supplementary Figures S2a and b, S3a-c and S4). Similarly, increase in survival and ATP level was detected in WT-MEFs grown in the presence of these substrates together with AICAR as compared with DKO MEFs (Figures 1g-i). Pharmacological inhibition of enzymes involved in the metabolism of lactate and glutamine by oxamate and 6-diazo-5-oxo-L-norleucine (DON) or epigallocatechin 3-gallate (EGCG), respectively, resulted in reduced cell survival even upon activation of AMPK (Supplementary Figures S2a-d).

Next to determine as to how AMPK facilitates cell survival in the presence of these substrates, we measured ATP level in the presence or absence of oligomycin, an ATP synthase inhibitor. Oligomycin reduced cell survival in the presence of non-glucose substrates, whereas under normal (glucose abundance) conditions oligomycin did not affect cell survival (Figures $1 \mathrm{~h}$ and j; Supplementary Figure S4), which is correlated with their respective ATP levels (Figures $1 \mathrm{i}$ and $\mathrm{k}$ ). These results suggest that AMPK is involved in promoting metabolism of non-glucose substrate to maintain energy homeostasis and cell survival.
AMPK induces mitochondrial biogenesis and OXPHOS activity under glucose-limiting conditions

For cancer cells to utilize non-glucose carbon sources such as lactate and glutamine, functional mitochondria are required. As AMPK regulates mitochondrial biogenesis in skeletal muscle upon chronic exercise or energy deprivation, ${ }^{16,20-22}$ we sought to explore whether AMPK is also involved in mitochondrial biogenesis in cancer cells. To confirm this, biochemical and genetic tools were utilized. We noticed that H1299-EV and WT-MEFs grown under glucose-limiting conditions showed high mitochondrial density as compared with those cultured in glucose-abundant conditions (Figures 2a-c). Both AICAR and rapamycin increased mitochondrial density in H1299-EV cells and WT-MEFs under glucose-limiting conditions (Figures 2a-c). However, we did not observe significant change in mitochondrial density in H1299-DN and AMPK-DKO grown under these conditions (Figures 2a-c). Also, increased mitochondrial density was detected in WT-MEF and H1299-EV cells treated with AICAR together with rapamycin as compared with single agent alone (Supplementary Figures S5a and b), whereas no such change was observed in DKO and H1299-DN cells grown under similar conditions (Supplementary Figures S5a and b).

Further, to confirm these observations, we checked citrate synthase and respiratory complex I activity. We found increased citrate synthase and complex I activity in H1299 cells under glucose-limiting conditions, which was further increased upon AICAR or rapamycin treatment (Figures $2 \mathrm{~d}$ and e). Under glucoselimiting conditions, decrease in ATP levels was detected that increased upon AICAR and rapamycin treatments (Figure 2f). Similar observations were also noted in WT-MEFs. However, in AMPK-DKO cells, irrespective of glucose concentrations as well as AICAR or rapamycin treatment, citrate synthase activity remained unchanged (Figure 2g). In WT-MEFs, relative ATP level was increased upon AICAR and rapamycin treatment under glucoselimiting conditions. However, in AMPK-DKO cells, only rapamycin increased the ATP level under similar conditions (Figure $2 \mathrm{~h}$ ).

Next, we checked molecular events involved in the mitochondrial biogenesis. Increased activation of AMPK and consequent alteration in phosphorylated ACC and p70-S6Kinase were detected in $\mathrm{H} 1299$ cells cultured in glucose-limiting conditions (Figure 2i). Under these conditions, pronounced elevation in the levels of proteins regulating mitochondrial biogenesis (PGC-1a and TFAM) was observed. AICAR treatment further increased the activation of AMPK and levels of PGC-1 $a$ and TFAM (Figure 2i). Interestingly, we also observed increased level of PGC-1a and TFAM in H1299 cells upon rapamycin treatment under both glucose-abundant and -limiting conditions (Figure 2i). These results indicate that AMPK maintains energy homeostasis under glucose-limiting conditions by promoting mitochondrial biogenesis.

AMPK-induced mitochondrial biogenesis is mediated by p38-dependent regulation of PGC-1a

As PGC-1 $a$ and TFAM are involved in mitochondrial biogenesis, we, therefore, explored the upstream events regulating these proteins. It is reported that p38 activates PGC- $1 a$, and AMPK is known to activate p38 under chronic energy deprivation. ${ }^{23-27}$ Thus, to investigate whether AMPK can activate p38 in cancer cells, we checked levels of phosphorylated p38 (p-p38) in H1299-EV, H1299-DN, WT-MEFs and AMPK-DKO cells grown under glucose-limiting conditions. Increased levels of p-p38 was detected in H1299-EV as well as in WT-MEFs exposed to low glucose or treated with AICAR. An increase in the levels of mitochondrial markers (PGC-1a, TFAM and COX IV) was also observed under similar conditions (Figures $3 a$ and $b$ ). On the contrary, reduced levels of phosphorylated p38, as well as PGC-1a, TFAM and COX IV were observed under similar conditions in 
a
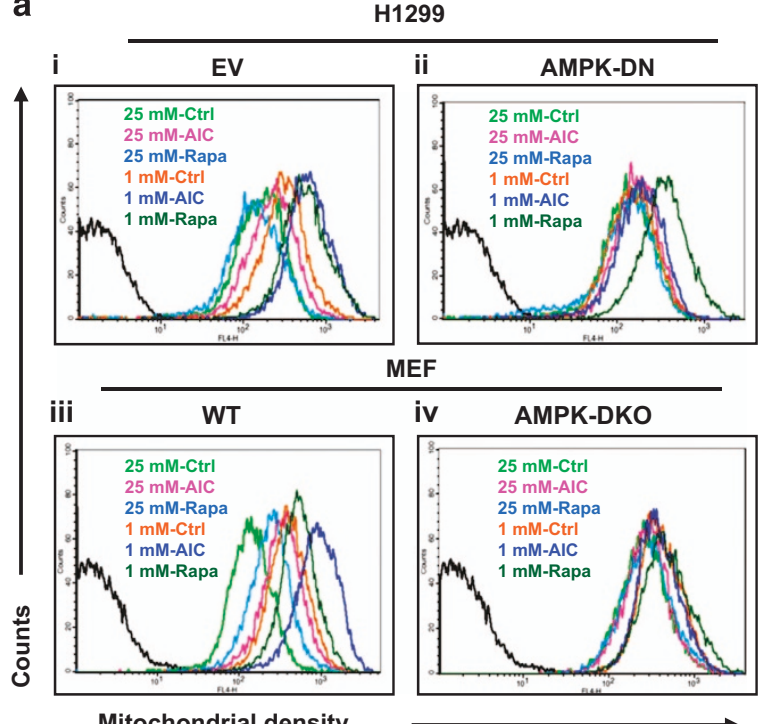

iv AMPK-DKO

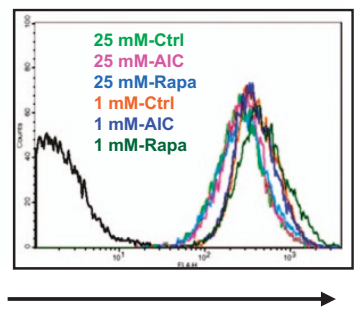

b

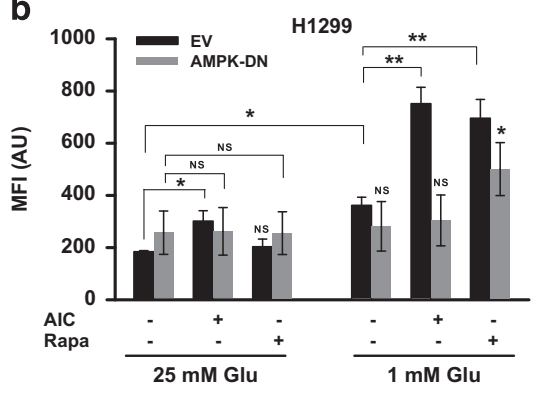

c

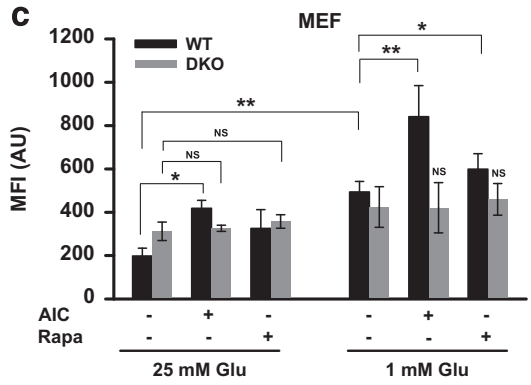

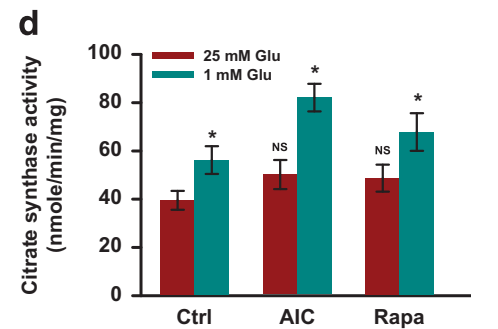

g

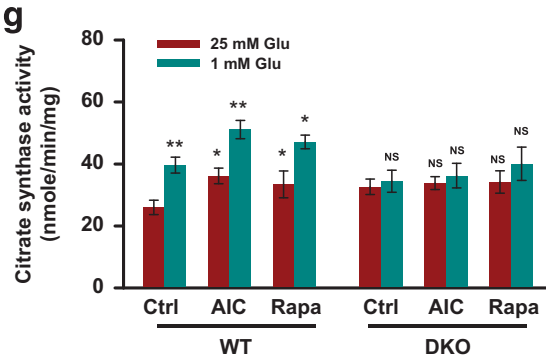

h

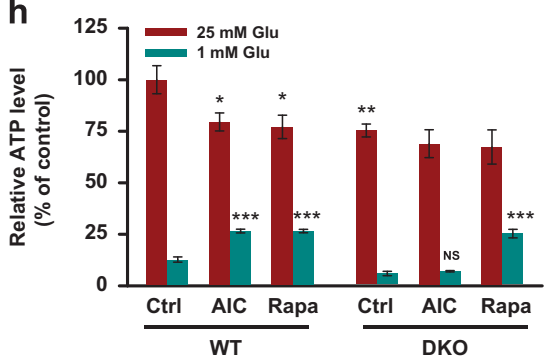

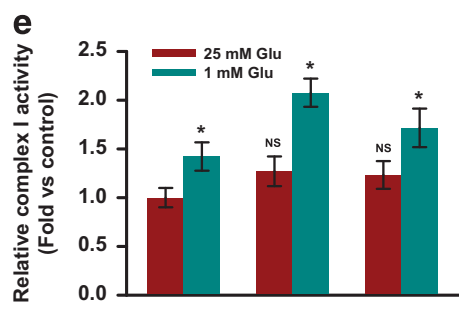

$\mathbf{f}$

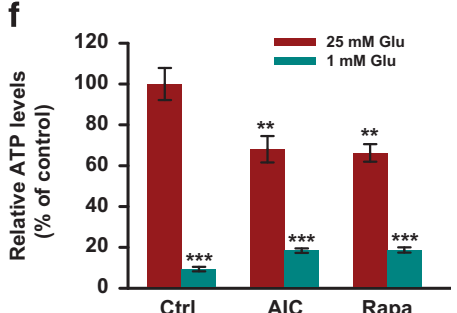

i

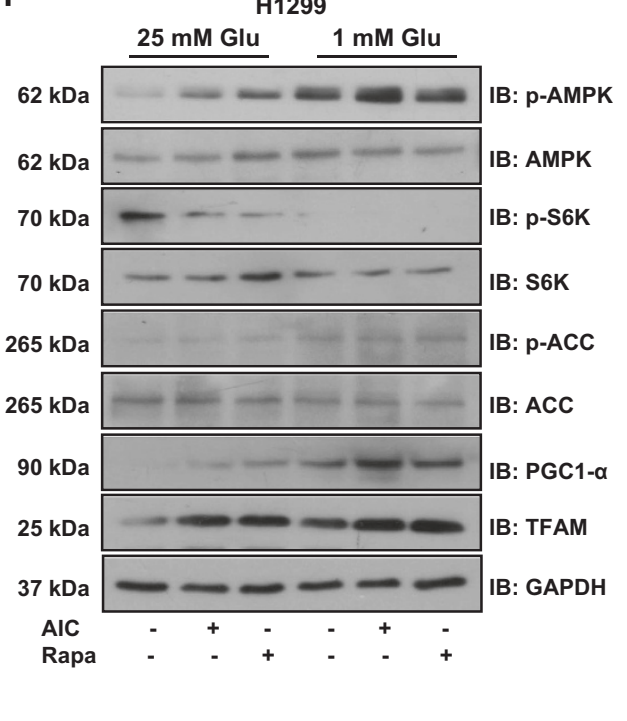

Figure 2. AMPK increases mitochondrial density in cancer cells. (a) H1299-EV (i), H1299-DN (ii), WT (iii) and AMPK-DKO (iv) cells were cultured in either $25 \mathrm{mM}$ or $1 \mathrm{mM}$ glucose, and $2 \mathrm{mM}$ glutamine in the presence of $0.5 \mathrm{mM}$ AICAR or $20 \mathrm{nM}$ rapamycin for $24 \mathrm{~h}$. Mitochondrial density was measured using MitoTracker Red FM by flow cytometry. $(\mathbf{b}, \mathbf{c})$ Quantitative bar graphs showing the relative change in the mean fluorescence intensity of MitoTracker Red FM in H1299 cells (b) and MEFs (c) under indicated treatment conditions. (d-f) Citrate synthase activity (d), respiratory complex I activity (e) and relative ATP level (f), in H1299 cells grown in DMEM containing 25 or $1 \mathrm{mM}$ glucose with or without AICAR and rapamycin for $24 \mathbf{h}$. (g, h) Citrate synthase activity (g) and relative ATP level (h), in WT and AMPK-DKO MEFs cultured under indicated conditions. (i) Immunoblots showing protein levels of indicated molecules involved in AMPK/mTOR pathway and mitochondrial biogenesis. Values are represented as mean \pm S.D. ${ }^{*} P<0.05,{ }^{* *} P<0.01$ and ${ }^{* * *} P<0.001$ denote significant differences between the groups. 


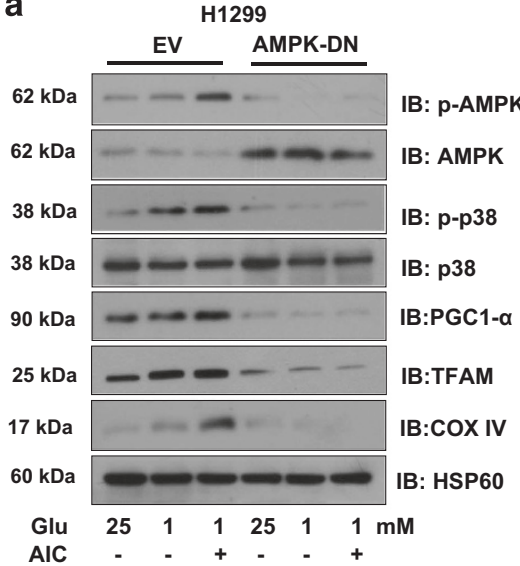

b

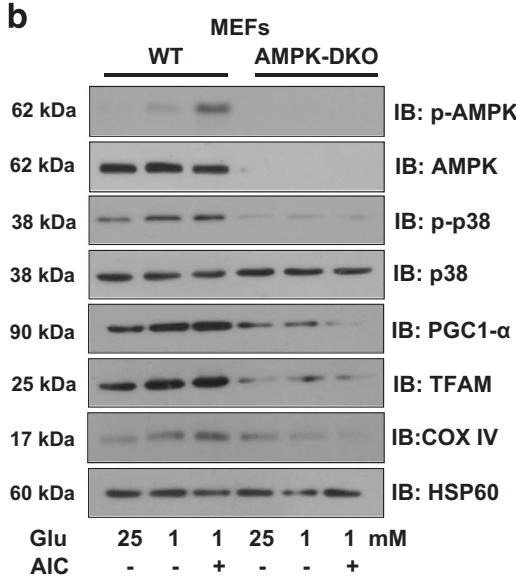

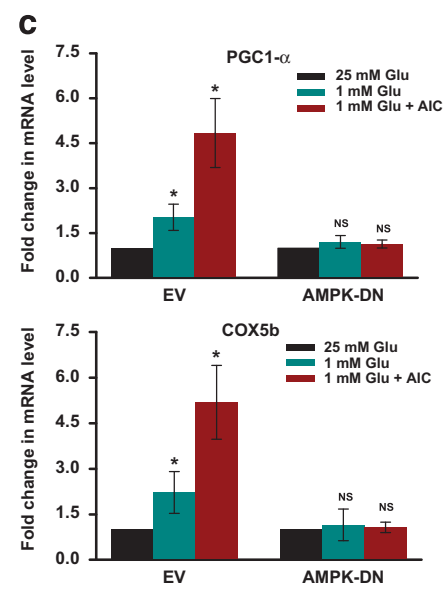

d
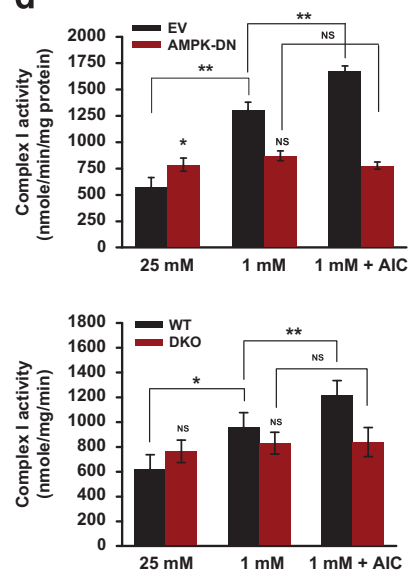

e
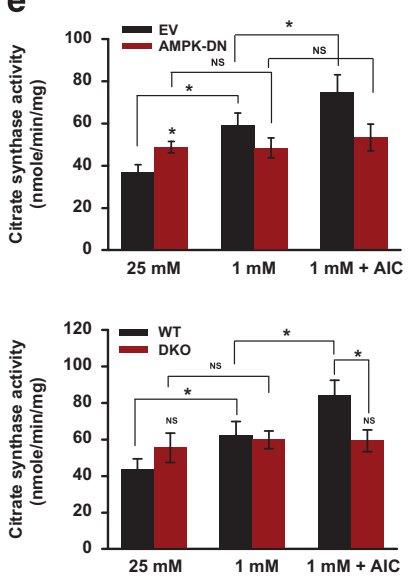

f
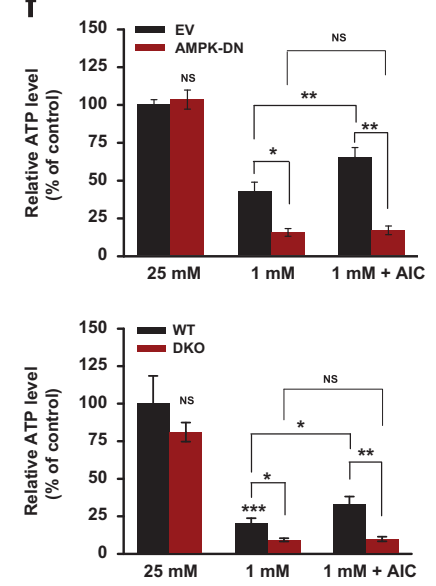

9

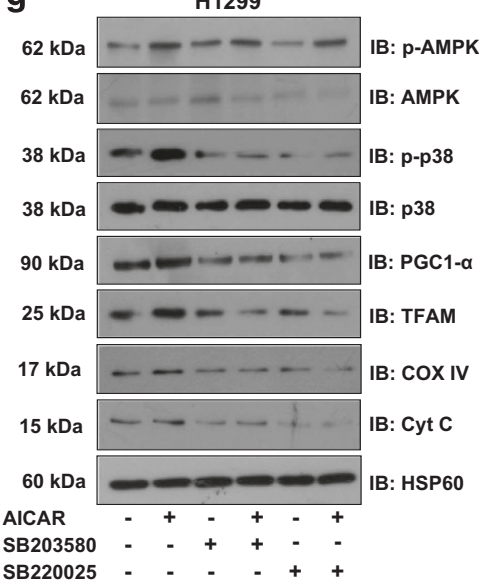

Figure 3. AMPK regulates p38-dependent expression of PGC-1a. $(\mathbf{a}, \mathbf{b})$ Immunoblots showing levels of indicated molecules in H1299-EV and H1299-DN cells (a) and in MEFs (b) cultured in 25 or $1 \mathrm{mM}$ glucose with or without $0.5 \mathrm{mM}$ AICAR for $24 \mathrm{~h}$. HSP60 was used as loading control. (c) Relative expression of PGC-1 $a$ and COX5b mRNA in H1299-EV and H1299-DN cells grown under the conditions used in a as evaluated by qRT-PCR. (d-f) H1299-EV, H1299-DN, WT-MEF and DKO cells were grown under conditions mentioned in a. Respiratory complex I activity (d), citrate synthase activity (e) and relative levels of ATP (f) were measured as described in Materials and Methods. (g) Immunoblots of indicated molecules in $\mathrm{H} 1299$ cells cultured in glucose-limiting conditions with or without $0.5 \mathrm{mM}$ AICAR and $10 \mu \mathrm{M}$ p38-specific inhibitors, SB203508 and SB220025 for $24 \mathrm{~h}$. Values are represented as mean \pm S.D. ${ }^{*} P<0.05,{ }^{* *} P<0.01$ and ${ }^{* * *} P<0.001$ denote significant differences between the groups.

H1299-DN and DKO-MEF cells (Figures 3a and b). Moreover, we noticed the increase in mRNA levels of PGC-1a and COX5b in H1299-EV and WT-MEF cells grown under glucose-limiting conditions or upon activation of AMPK as compared with their respective counterparts (Figure $3 \mathrm{c}$ ). Under glucose-limiting conditions, activities of respiratory complex I and citrate synthase were increased, which were further elevated by AICAR in H1299-EV and WT-MEFs (Figures 3d and e). However, irrespective of glucose concentration and AICAR treatment, activity of these enzymes remained unaltered in H1299-DN and AMPK-DKO cells (Figures 3d and e). Relative ATP level was increased upon AICAR treatment in H1299-EV and WT-MEFs under glucose-limiting conditions, which was unaffected in H1299-DN and AMPK-DKO cells (Figure 3f).

To confirm whether $\mathrm{p} 38$ is involved in the regulation of PGC-1a, we used p38-specific inhibitors SB203580 and SB220025. We observed that both these inhibitors diminished phosphorylation of p38 though the level of p-AMPK was unaffected in H1299 cells. Reduced levels of PGC-1 $a$, TFAM, COX IV and Cyt C were detected in H1299 cells treated with these inhibitors even upon activation of AMPK (Figure 3g). Moreover, we noted that inhibition of p38 caused cell death under metabolic stress condition as evident by reduced ATP level in $\mathrm{H} 1299$ cells treated with AICAR and p38 inhibitors (Supplementary Figures S6a-d), which is likely because of disruption in energy homeostasis mediated by AMPK. To verify whether AMPK-induced activation of p38 is mediated by mTOR, we checked phosphorylated p38 level in $\mathrm{H} 1299$ cells grown under glucose-limiting conditions in the presence or absence of p38 inhibitor SB203580. We observed that rapamycin treatment did not influence the level of phosphorylated p38 (Supplementary Figure S7), though the levels of molecules involved in mitochondrial biogenesis were increased (Supplementary Figure S7), suggesting that AMPK-mediated activation of p38 is independent of mTOR. Collectively, these results suggest that activation of p38 is required for the AMPK-dependent mitochondrial biogenesis.

AMPK controls metabolic adaptation under glucose limitation To explore whether AMPK is involved in maintaining metabolic and energy homeostasis, we cultured MEF cells in 25 or $1 \mathrm{mM}$ glucose for $24 \mathrm{~h}$, and these were replenished with $25 \mathrm{mM}$ glucose thereafter. We noticed that AMPK-DKO cells are larger in size as compared with WT cells cultured in $25 \mathrm{mM}$ glucose (Figure $4 \mathrm{a}-\mathrm{i}$ ). An increase in the cell size of WT-MEF was observed upon switching them to $1 \mathrm{mM}$ glucose, while replenishing glucoserestored cell size (Figure 4a-ii). On the other hand, cell size did not 
a

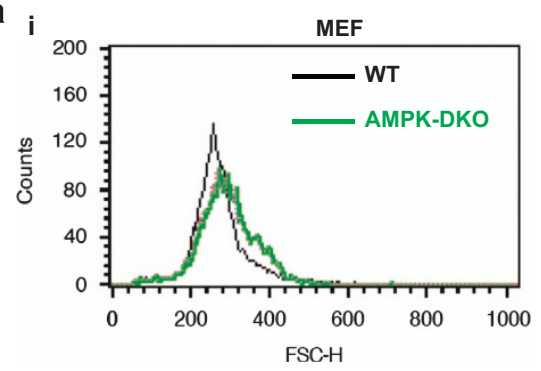

b

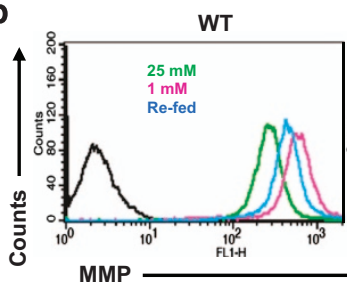

C

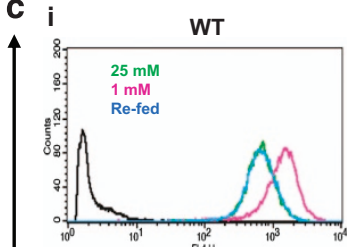

ii AMPK-DKO
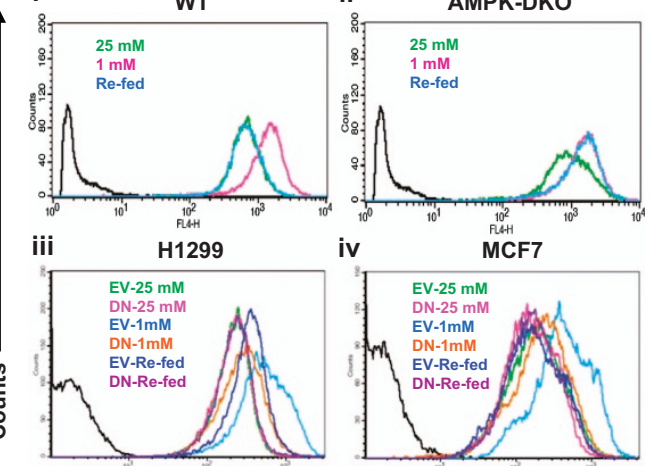

iv
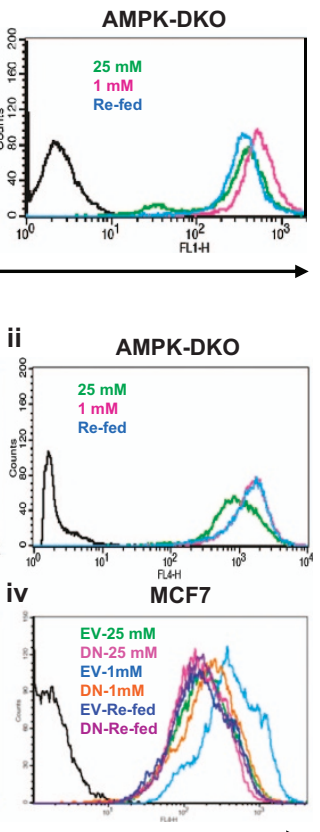

ii

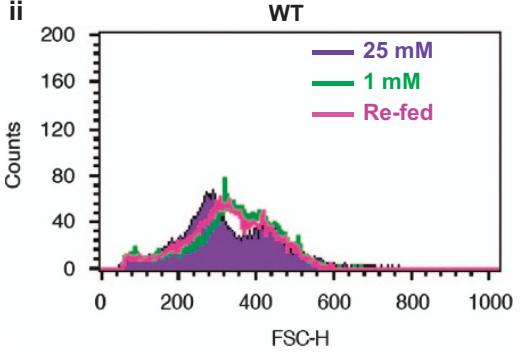

d

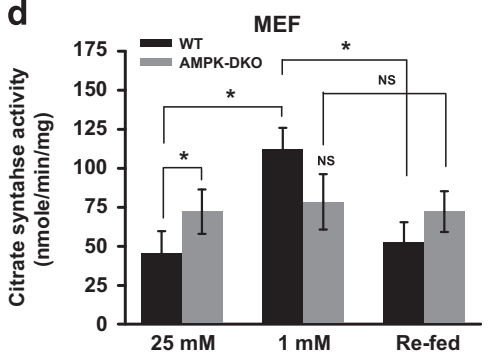

f

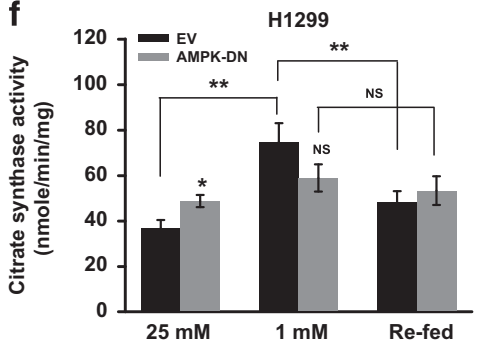

iii

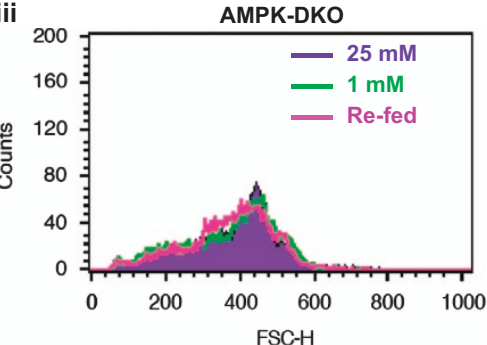

Mitochondrial Density

Figure 4. AMPK controls metabolic homeostasis in cancer cells. (a) WT and DKO MEFs were cultured in DMEM containing either 25 or 1 mM glucose and $2 \mathrm{mM}$ glutamine for $24 \mathrm{~h}$. The medium was replaced with fresh medium containing $25 \mathrm{mM}$ glucose and further grown for $24 \mathrm{~h}$. Size of these cells were determined by flow cytometry. Relative size of WT and AMPK-DKO cells under normal metabolic condition ( 25 mM glucose) (i), change in cell size of WT-MEF (ii) and DKO-MEF (iii), cultured in the presence of $25 \mathrm{mM}$ glucose, $1 \mathrm{mM}$ glucose or re-fed with $25 \mathrm{mM}$ glucose. (b) Mitochondrial membrane potential (MMP) in MEF cells grown under the conditions mentioned in a. DiOC6 was used to determine MMP by flow cytometry. (c) MEF and cancer cells (H1299-DN and MCF7-DN) were grown under the conditions mentioned above. Mitochondrial density was determined by flow cytometry using probe MitoTracker Red FM. Mitochondrial density in WT (i), AMPK-DKO MEFs (ii), H1299 (EV and DN) (iii) and MCF7 (EV and DN) cells (iv). (d-f) MEFs (WT and DKO), H1299 (EV and DN) cells were cultured under the indicated conditions for $24 \mathrm{~h}$. Citrate synthase activity (d and $\mathbf{f}$ ) and relative ATP levels (e and $\mathbf{g}$ ) were determined in these cells as described in Materials and Methods. Values are represented as mean \pm S.D. ${ }^{*} P<0.05$ and ${ }^{* *} P<0.01$ denote significant differences between the groups.

alter in DKO cells under identical conditions (Figure 4a-iii). This observation suggests the involvement of AMPK in regulating cell size under metabolic stress.

Subsequently, a prominent increase in mitochondrial membrane potential (MMP) and density was observed in WT cells under glucose-limiting conditions (Figures 4b (left) and c-i), which was restored upon glucose replenishment (Figures $4 \mathrm{~b}$ (left) and c-i). AMPK-DKO cells exhibited an increase in MMP and mitochondrial density even under glucose-abundant conditions while a moderate increase in mitochondrial density was observed under glucose-limiting conditions (Figures $4 \mathrm{~b}$ (right) and c-ii). However, upon replenishing glucose to DKO cells cultured in glucose-limiting conditions, MMP and mitochondrial density were not restored (Figures $4 \mathrm{~b}$ (right) and c-ii). Similar findings were also observed in H1299 and MCF7 cells stably transfected with either empty vector or DN-AMPK (Figures 4c-iii and c-iv). Moreover, enhanced mitochondrial citrate synthase activity was detected in WT-MEFs and H1299-EV cells under glucose-limiting conditions, which decreased upon replenishing glucose (Figures $4 d$ and f). However, no significant change was observed in citrate synthase activity in AMPK-DKO and H1299-DN cells (Figures 4d and f). Further, we checked whether cells are able to maintain ATP level under the condition of fluctuating glucose concentrations. We found that relative ATP levels were decreased in WT-MEF and H1299-EV cells under glucose-limiting conditions, which was increased upon replenishing glucose (Figures $4 \mathrm{e}$ and g). In AMPKDKO and H1299-DN cells, glucose-limiting condition caused a reduction in the ATP level. Upon glucose replenishment, however, there was relatively slight increase in ATP level (Figures $4 \mathrm{e}$ and g).

AMPK is known to induce glycolysis by directly phosphorylating 6-phosphofructo-2-kinase (PFK2) in muscles, which by activating PFK, enhances glycolytic rate. ${ }^{28,29}$ To explore role of AMPK in metabolic homeostasis, we cultured $\mathrm{H} 1299$ cells in the presence of varying concentrations of glucose with or without AICAR and compound $\mathrm{C}$, and after $24 \mathrm{~h}$, medium was replenished with fresh medium containing $25 \mathrm{mM}$ glucose. We noticed that cells grown 
$\mathbf{a}_{\mathrm{i}}$

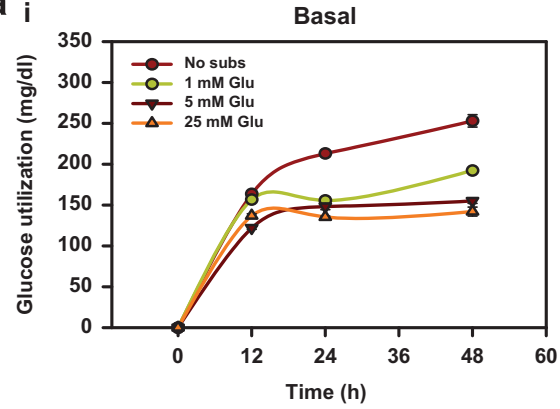

ii

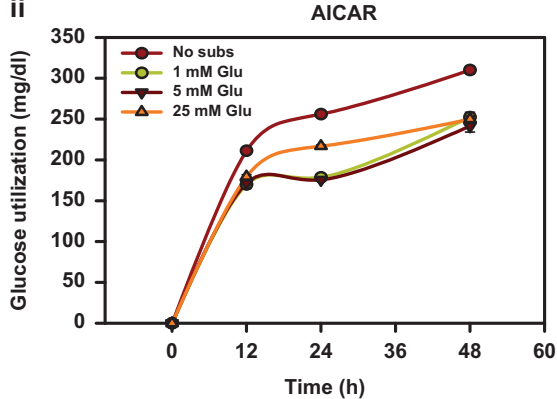

iii

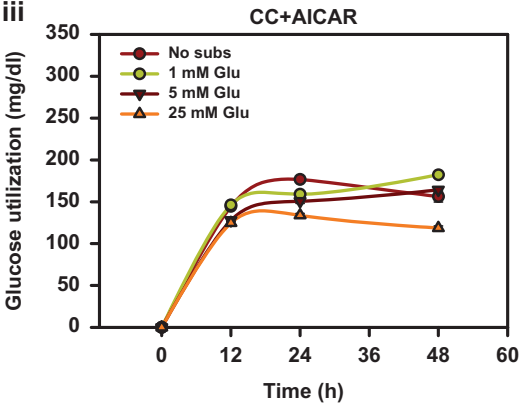

b

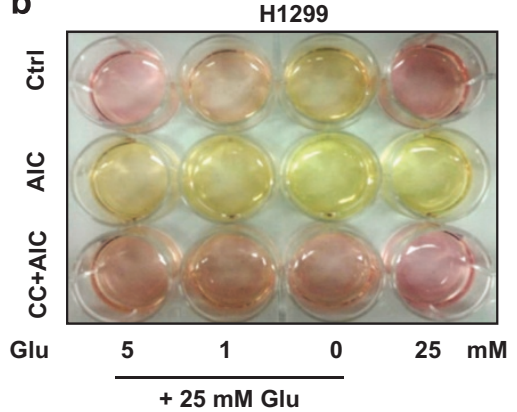

e

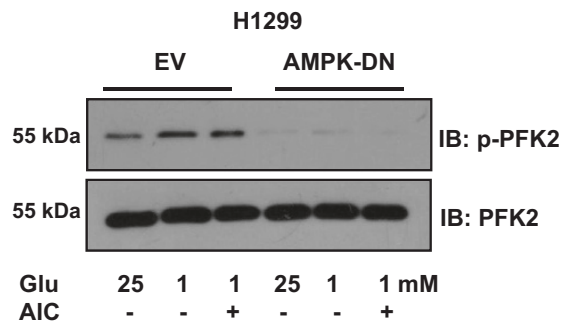

C

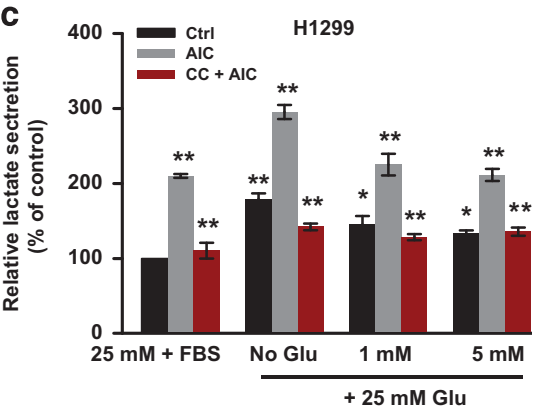

f

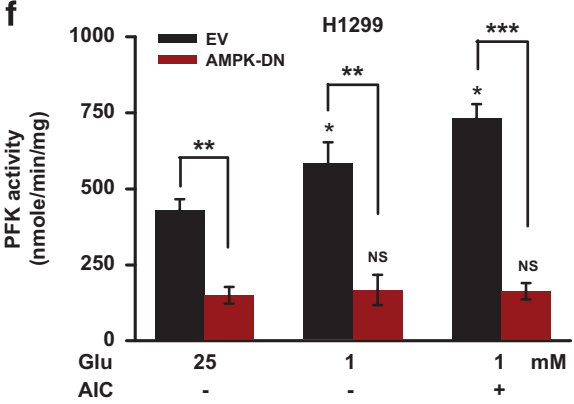

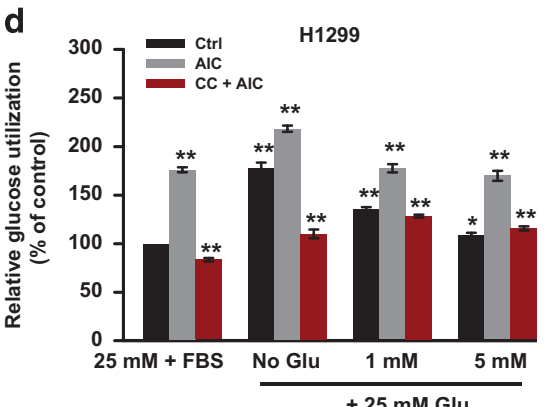

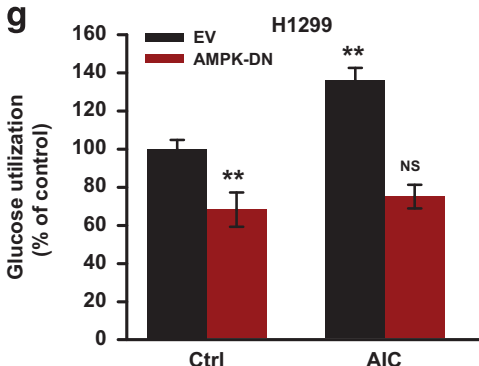

Figure 5. AMPK promotes glycolysis by activating PFK2. (a) $\mathrm{H} 1299$ cells were grown in DMEM without glucose and glutamine, and supplemented with $1 \mathrm{mM}, 5 \mathrm{mM}$ and $25 \mathrm{mM}$ glucose. Cells were simultaneously treated with $0.5 \mathrm{mM}$ AICAR and/or $10 \mu \mathrm{M}$ compound C for $24 \mathrm{~h}$. Fresh medium (DMEM containing $25 \mathrm{mM}$ glucose and 10\% FBS) was added after $24 \mathrm{~h}$ and further grown for indicated time points. Timedependent glucose utilization in $\mathrm{H} 1299$ cells under basal (i), AICAR treated (ii) or in the presence of compound C plus AICAR (iii). (b) Representative photographs of culture medium from $\mathrm{H} 1299$ cells grown under above-mentioned conditions for $24 \mathrm{~h}$. (c, d) lactate secretion (c) and glucose utilization (d) in $\mathrm{H} 1299$ cells grown under the conditions used in a. (e) Immunoblots showing phosphorylated and basal levels PFK2 in H1299 (EV and DN) cells grown under indicated conditions for 24 h. (f) PFK activity in H1299 (EV and DN) cells grown under indicated conditions for $24 \mathrm{~h}$. (g) Relative glucose utilization in H1299-EV and H1299-DN cells treated with or without AICAR for $36 \mathrm{~h}$. Values are represented as mean \pm S.D. ${ }^{*} P<0.05,{ }^{* *} P<0.01$ and ${ }^{* *} P<0.001$ denote significant differences between the groups.

in no or less glucose exhibited increased glucose utilization in a time-dependent manner upon replenishing glucose (Figure 5a-i). AICAR treatment further enhanced glucose utilization which was reduced by compound $\mathrm{C}$ under these conditions (Figures 5a-ii and a-iii). Lactate secretion (Figures $5 b$ and c) was proportionally associated with utilization of glucose under these conditions (Figure 5d). Increased phosphorylation of PFK2 with concomitant increased activity of rate-limiting enzyme PFK was detected in H1299-EV cells grown in glucose-limiting conditions and/or treated with AICAR (Figures $5 \mathrm{e}$ and $\mathrm{f}$ ). However, no change in the phosphorylated level of PFK2 and PFK activity was observed in H1299-DN cells under identical conditions (Figures $5 e$ and $f$ ). A significant decrease in the glucose utilization was observed in H1299-DN cells treated or untreated with AICAR as compared with H1299-EV cells (Figure $5 \mathrm{~g}$ ). Our results suggest that AMPK mediates metabolic and energy homeostasis in cancer cell under glucose-limiting conditions by promoting both glycolytic as well as mitochondrial metabolism.

\section{DISCUSSION}

AMPK, under nutritional stress, maintains energy balance by minimizing energy expense and accelerating ATP generation to restore its level in favor of cell survival. ${ }^{30}$ Role of AMPK in cancer is not very clear as it has been reported to exhibit both tumorsuppressing as well as tumor-promoting function. ${ }^{31-33}$ Considering the heterogeneity and physiological stress in tumor microenvironment, it is inevitable for cancer cells to evolve a mechanism to overcome/adapt to various stresses to maintain survival. Our study highlights the important role of AMPK in maintaining energy and metabolic homeostasis, and in promoting cell survival under glucose-limiting conditions.

Role of AMPK in cell survival and metabolic homeostasis in normal (noncancerous) tissues has already been well understood. ${ }^{11}$ However, its role in cancer remains paradoxical. Jeon et al. $^{33}$ have shown that activation of AMPK is required for the survival of cancer cells by maintaining redox balance and by promoting fatty acid oxidation. It has also been shown that mTOR 
inhibition prolongs cell survival under glucose-deprived conditions in TSC2 null cells. ${ }^{19}$ Consistent with these reports, our results demonstrate that AMPK-mediated cell survival requires inhibition of mTOR, as rapamycin promotes survival under both AMPK-activated or -inactivated conditions. Under glucose-limiting conditions, activation of mTOR would perturb energy homeostasis as mTOR mainly regulates anabolic processes. ${ }^{19}$ Thus, inhibition of mTOR is essential to maintain energy and metabolic homeostasis under metabolic stress. Our data show that mTOR inhibition augments ATP levels which positively correlate with cell survival even in the absence of AMPK. As AMPK and mTOR have antagonistic role in cells, inhibition of mTOR might be required for AMPK-mediated metabolic homeostasis. Importantly, we show that AMPK facilitates metabolism of non-glucose substrates, specially glutamine and lactate, to maintain cell survival. This could be an alternative mechanism to restore cellular ATP level under glucose-limiting conditions. It has been shown that in the absence of glucose, glutamine serves as a major energy source in cancer cells. ${ }^{19,34}$ Similarly, cancer cells can also metabolize lactate and fatty acids, under metabolic stress, to derive ATP. ${ }^{33,35}$ Our results demonstrate that activation of AMPK is required for the metabolism of these substrates as cells lacking functional AMPK fail to grow in the presence of these substrates. This alternative metabolism can provide survival advantage to cancer cells under glucose-limiting conditions.

To generate more ATP, enhanced mitochondrial function is required. It has been reported that, under chronic energy deprivation, mitochondrial activity is enhanced in muscle cells. ${ }^{17,22}$ CPT1C, a mitochondrial enzyme, has been shown to maintain survival of breast cancer cells treated with metformin under metabolic stress. ${ }^{36}$ Interestingly, mitochondrial biogenesis and its function are elevated in response to chronic energy deprivation in muscle. ${ }^{17}$ In agreement with these findings, we report that mitochondrial activity is enhanced in cancer cells under glucose-limiting conditions. Recent correlative studies suggest that activation of AMPK is associated with increase in levels and activity of mitochondrial enzymes ${ }^{16}$ and also in mitochondrial biogenesis, in rat skeletal muscles. Concurrent with these reports, we observed that enhanced mitochondrial biogenesis and activation of AMPK is required for maintaining mitochondrial OXPHOS capacity to sustain ATP levels in cancer cells under metabolic stress. Our results indicate that activation of AMPK enhances expression of PGC-1a and its target genes in cancer cells under metabolic stress. PGC-1a is a major transcription coactivator protein required for mitochondrial biogenesis. PGC-1a promotes oxidative metabolism, mitochondrial biogenesis and facilitates glutamine metabolism to support proliferation ${ }^{37-39}$ and metastasis, ${ }^{4}$ although very little is known about the regulation of PGC-1a in cancer cells. Our data suggest that expression of PGC-1a can be regulated by AMPK. Previously, it has been reported that rapamycin increases expression of PGC $-1 a^{40}$ We also observed that rapamycin treatment increases expression of the PGC-1a independent of AMPK suggesting that mTOR inversely correlates with PGC-1a expression and mitochondrial biogenesis. It is likely that AMPK could regulate mitochondrial biogenesis via inhibition of mTOR. In addition, we demonstrate that activation of AMPK with concomitant inhibition of mTOR is a prerequisite for maintaining cellular ATP level as well as mitochondrial function.

Stress kinase p38MAPK has been shown to regulate mitochondrial biogenesis by regulating expression of PGC- $1 a$ in muscles ${ }^{24,25}$ and AMPK can activate p38. ${ }^{41}$ The role of both AMPK and p38 in tumorigenesis is a paradox. ${ }^{42-44}$ Activation of both these kinases has been shown to inhibit cell proliferation. ${ }^{31,45}$ On other hand, it has also been reported that p38 signaling cascades augment cell survival and growth. ${ }^{46,47}$ Our findings indicate that p38 activity is required for AMPK-mediated cell survival under metabolic stress; and as these two together can regulate PGC- $1 a$, we sought to explore the connection between the two. Our results suggest that p38 is a downstream activator of AMPK-induced PGC-1 $a$ expression, and inhibition of p38 does not affect AMPK activation but reduces the levels of PGC-1 $a$ and TFAM. As both of these are stress kinases, we reasoned that increased activity of these kinases is required to maintain cancer cell survival under glucose-limiting conditions.

Adaptation to metabolic changes and nutrient availability in tumor microenvironment is an important biological process for cell survival. $^{32,33}$ Our data show that cells respond to the nutrient availability by regulating mitochondrial activity which increases under limited glucose availability and can be restored to normal levels upon re-feeding cells with glucose. Interestingly, inhibition of AMPK abrogates cells ability to adapt to fluctuation in glucose levels thereby perturbing metabolic/energy homeostasis leading to cell death. Another important aspect of metabolic homeostasis is to speed up the rate of metabolic reaction to regenerate more ATP and increase in glycolysis under metabolic stress is considered as an adaptive response of cells for maintaining energy homeostasis. ${ }^{28,29}$ We also noted that, AMPK by phosphorylating PFK2, enhances glycolysis. AMPK-dependent increase in glycolysis is a homeostatic mechanism to rapidly restore the energy pool of the cells under metabolic stress in heart and muscle. ${ }^{28,29}$

Conclusively, above-stated results indicate that AMPK is an important proximal signaling step for regulating mitochondrial biogenesis and cell survival under metabolic stress. We, for the first time, show that AMPK can regulate mitochondrial biogenesis in cancer cells to promote metabolism of non-glucose carbon sources via regulating p38/PGC-1a (Figure 6). Together, our study

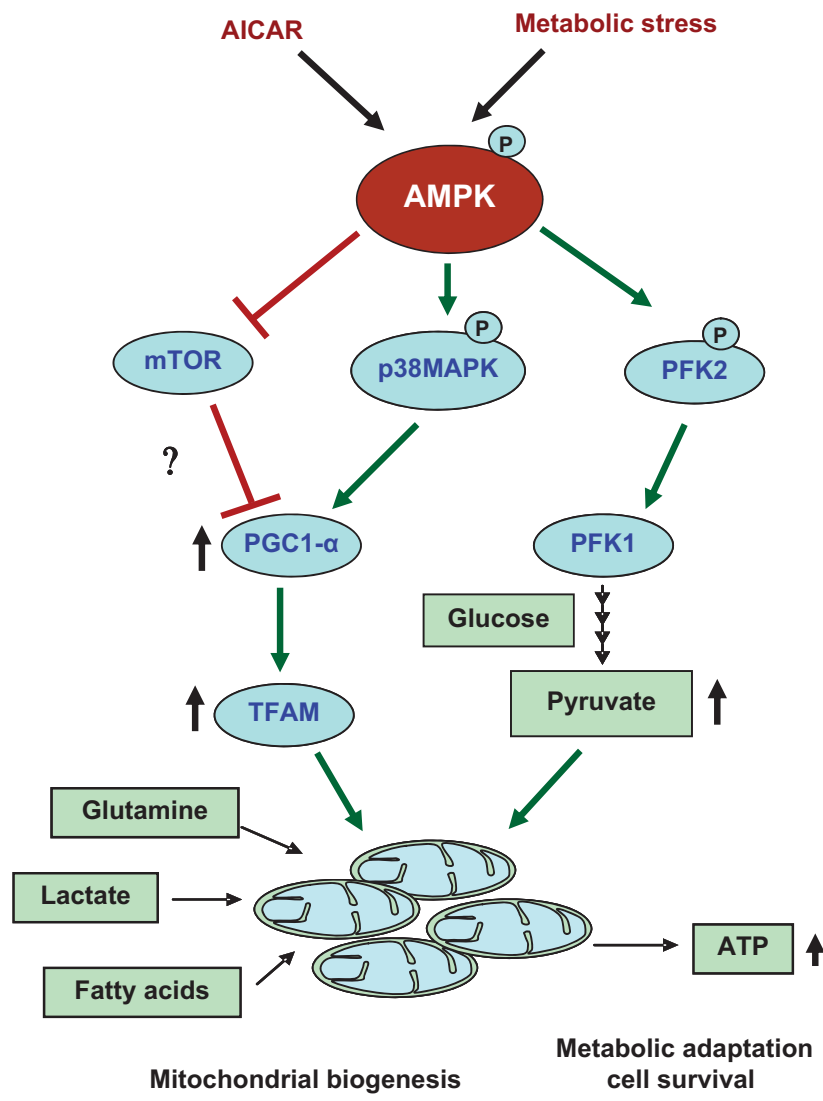

Figure 6. Schematic representation of the mechanism of AMPKmediated cell survival. AMPK is activated under glucose-limiting conditions as well as by AICAR. AMPK, by increasing p-p38 and by inhibiting mTOR, regulates expression of PGC- $1 a$ which controls mitochondrial biogenesis in cancer cells and allows oxidative metabolism of non-glucose carbon sources (glutamine, lactate and fatty acids) to generate ATP. To restore ATP pool, AMPK enhances metabolic rate by activating PFK2. 
provides mechanistic insight into the protective role of AMPK in cancer cells under glucose-limiting conditions.

\section{MATERIALS AND METHODS}

Cell lines and culture conditions

H1299 and MCF7 cells were obtained from ATCC (Manassas, VA, USA) and maintained in our in-house repository. H1299 cells expressing dominantnegative (AMPK-DN) form of AMPK- $a 1$ subunit were generated by transfecting pCDNA3-AMPKa1-DN or empty vector (pCDNA3) only. AMPK- $a 1 / a 2^{+/+}$(WT) and AMPK- $a 1 / a 2^{-/-}$(DKO) SV40-immortalized MEFs have been described previously. ${ }^{32}$ All the cells were grown in DMEM containing $2 \mathrm{mM} / \mathrm{l}$ glutamine supplemented with $10 \%$ FBS and $1 \%$ penicillin/streptomycin (Life Technologies, Carlsbad, CA, USA). For experimental purpose, culture conditions were defined as, (i) glucose-abundant condition: $25 \mathrm{mM}$ glucose and 10\% FBS; (ii) glucose-limiting condition: $1 \mathrm{mM}$ glucose, $2 \mathrm{mM}$ glutamine and $10 \%$ FBS; (iii) glucose-deprived condition: $2 \mathrm{mM}$ glutamine and 1\% FBS.

\section{Chemicals and reagents}

AICAR, compound $C$, glucose, glutamine, palmitic acid (sodium salt), lactate, oligomycin, rapamycin, 6-diazo-5-oxo-L-norleucine (DON) and epigallocatechin 3-gallate (EGCG) were purchased from Sigma (St. Louis, MO, USA). SB203580 and SB220025 were obtained from Calbiochem (San Diego, CA, USA). Antibodies for p-AMPK-Thr 172 (Cat\# 2535, Dil 1:1000), p-p70S6K-Thr 389 (Cat\# 9234, Dil 1:1000) and p-p38-Thr 180/Tyr 182 (Cat\# 9211, Dil 1:1000) were purchased from Cell Signaling Technology (Danvers, MA, USA). Antibodies for pACC-Ser 79 (Cat\# SC271965, Dil 1:1000), ACC (Cat\# SC30212, Dil 1:1000), p70-S6Kinase (Cat\# SC230, Dil 1:1000), AMPK (Cat\# SC25792, Dil 1:1000), PGC-1a (Cat\# SC13067, Dil 1:1000), TFAM (Cat\# SC376672, Dil 1:2000), p-p38-Tyr 172 (Cat\# SC7973, Dil 1:1000), p38 (Cat\# SC535, Dil 1:1000), Survivin (Cat\# SC17779, Dil 1:1000), Bcl-2 (Cat\# SC7382, Dil 1:1000), Bcl-XL (Cat\# SC7195, Dil 1:1000), COX IV (Cat\# SC292052, Dil 1:1000), Cyto C (Cat\# 7159, Dil 1:1000), pPFK2Ser 466 (Cat\# SC32966, Dil 1:1000), PFK2 (Cat\# SC10090, Dil 1:1000), c-Myc (9E10) (Cat\# SC40, Dil 1:1000), HSP60 (Cat\# SC13115, Dil 1:2000), GAPDH (Cat\# SC20357, Dil 1:1000) were from Santa Cruz Biotechnology (Dallas, TX, USA).

Determination of cell viability and cell size

Cell viability was assessed as described earlier by propidium iodide (PI) staining using flow cytometry. ${ }^{19}$ Briefly, cells were washed, trypsinized and pellet was collected by centrifuging at $1000 \times g$ for $5 \mathrm{~min}$. After washing with sterile PBS, the cells were incubated with PI $(200 \mathrm{ng} / \mathrm{ml}$ in PBS) for 10 min. PI fluorescence was acquired by flow cytometer using FL2 filter (FACS Calibur, BD Bioscience, San Jose, CA, USA). Cells positive with PI were counted as dead cells. Data were analyzed using Cell Quest Pro software (Becton Dickinson, San Jose, CA, USA) for $2.5 \times 10^{4}$ cells. The experiments were performed in triplicate and repeated at least once. Alternatively, cell number was counted using hemocytometer for accessing cell proliferation. Change in cell size was determined by measuring forward scatter (FSC) via flow cytometry as described previously. ${ }^{19}$

Long-term cell survival assay

Cells were seeded at a density of $1 \times 10^{3} /$ well in 12 -well culture plates, and were allowed to adhere for $24 \mathrm{~h}$ at $37^{\circ} \mathrm{C}$. Cells were treated as per the experimental requirement. Medium was replaced with fresh medium and further grown for 1 week. Crystal violet staining was performed as described. ${ }^{48}$

\section{siRNA transfection}

siRNA against AMPK- $\alpha 1 / 2$ and AMPK- $\alpha 1$ were purchased from Santa Cruz Biotechnology. Transfection was done using Lipofectamine 2000 (Life Technologies) according to the manufacturer's instructions. Transfection efficiency was assessed by simultaneously transfecting pEGFPN1 plasmid. Immunoblotting was performed to ensure inhibition of respective gene expression.
Cell lysate preparation and immunoblotting

Cell lysates were prepared and subjected to immunoblotting as described previously. ${ }^{48}$ Whenever required, membranes were washed thoroughly with TBS (Tris-buffered saline) and re-probed with desired antibodies.

\section{Quantitative real-time PCR analysis}

RNA from cells was extracted using TRIzol reagent (Invitrogen, Carlsbad, CA, USA) according to the manufacturer's instructions. CDNA was prepared using moloney murine leukemia virus reverse transcriptase (MMLV-RT) under the recommended conditions. qRT-PCR was performed on CDNA samples using the Quantitative SYBR green PCR kit (Bio-Rad, Hercules, CA, USA) and was run on the Mastercycler ep realplex Real-time PCR System (Eppendorf, Hamburg, Germany) as described earlier. ${ }^{4}$ Primer sequences used are listed in Supplementary Table S1.

\section{ATP measurement}

ATP level was measured in cells using commercially available ATP bioluminescence kit (Roche, Mannheim, Germany). Briefly, cells were grown in the presence of indicated drugs with or without lactate $(10 \mathrm{mM})$, glucose $(1,5$ and $25 \mathrm{mM})$, glutamine $(5 \mathrm{mM})$, galactose $(5 \mathrm{mM})$ and palmitate $(100 \mu \mathrm{M})$. Cells were collected and lysed in $100 \mathrm{mM}$ Tris-EDTA (TE) buffer containing $0.01 \%$ NP-40 and boiled for 1 min followed by three quick freeze-thaw cycles. Luminescence was recorded for blank as well as for samples. The experiments were done in triplicate and repeated at least twice, and the final values were normalized with total protein contents.

Mitochondrial density and membrane potential measurement For measuring membrane potential and mitochondrial density, the cells were resuspended in PBS containing either $100 \mathrm{nM}$ DiOC6 or $50 \mathrm{nM}$ MitoTracker Red FM (Life Technologies), respectively, and incubated for $30 \mathrm{~min}$ at $37^{\circ} \mathrm{C}$. The fluorescence of DiOC6 and MitoTracker Red FM were acquired through a $585 \mathrm{~nm}$ (FL1) or 650 (FL4) filter in a flow cytometer. Data were analyzed using Cell Quest Pro software (Becton Dickinson) for $1 \times 10^{4}$ cells.

\section{Glucose utilization and lactate estimation assay}

Cells $\left(3 \times 10^{5}\right)$ were cultured in DMEM containing 1 or $25 \mathrm{mM}$ glucose. After $24 \mathrm{~h}$, medium was replaced with respective medium containing AICAR or compound $\mathrm{C}$ for $24 \mathrm{~h}$, and residual glucose present in the spent medium was monitored using GOD-POD-based glucose assay kit (Spinreact, Girona, Spain). Consumed glucose was estimated by subtracting the remaining glucose in the medium from the initial concentration in control medium $(450 \mathrm{mg} / \mathrm{dl})$. All the experiments were performed in triplicate and the values were normalized to total number of cells. Lactate was estimated by using commercially available lactate estimation kit (Spinreact) according to the manufacturer's protocol. Briefly, cells $\left(3 \times 10^{5}\right)$ were plated in 12-well tissue culture plates. After $24 \mathrm{~h}$, medium was replaced with DMEM containing $0.2 \%$ BSA with $0,1,5$ and $25 \mathrm{mM}$ glucose either containing $0.5 \mathrm{mM}$ AICAR or $10 \mu \mathrm{M}$ compound $\mathrm{C}$ alone or together for $24 \mathrm{~h}$. The cells were washed with fresh medium and replaced with DMEM containing $25 \mathrm{mM}$ glucose in each well with or without AICAR and compound C for further $48 \mathrm{~h}$. The medium was collected and stored in $-20^{\circ} \mathrm{C}$ for assay purpose.

\section{Enzyme assays}

Enzyme activity was measured in cells as described earlier with slight modifications. ${ }^{49}$ Briefly, cells cultured under desired experimental conditions were homogenized in hypotonic $(20 \mathrm{mM})$ potassium phosphate buffer ( $\mathrm{pH}$ 7.5) containing protease inhibitor cocktail (Roche), vortexed and lysed by three cycles of freeze and thaw procedure. Activity of complex I and citrate synthase was determined as described. PFK activity was determined by enzyme-coupled reaction method. Briefly, the enzyme reaction was performed in Tris- $\mathrm{Cl}$ buffer $(50 \mathrm{mM}, \mathrm{pH}$ 8.0) containing ATP $(0.1 \mathrm{mM}), \mathrm{MgCl}_{2}(3.3 \mathrm{mM}), \mathrm{NADH}(0.1 \mathrm{mM})$, glyceraldehydes-3-phosphate dehydrogenase $(1 \mathrm{U} / \mathrm{ml})$, aldolase $(1 \mathrm{U} / \mathrm{ml})$, triose phosphate isomerase $(6 \mathrm{U} / \mathrm{ml})$. The reaction was started by adding $3.3 \mathrm{mM}$ fructose 6 -phosphate and absorbance was recorded at $340 \mathrm{~nm}$ at every $30 \mathrm{~s}$ for $10 \mathrm{~min}$. The values were normalized with protein contents. 
Statistical analysis

Unpaired two-tailed Student's $t$-tests were performed unless otherwise mentioned, using SPSS Software V.12 (San Jose, CA, USA). Data were represented as mean \pm S.D. unless otherwise specified. Experiments were done in triplicate and most of the experiments were repeated at least once. ${ }^{*} P<0.05,{ }^{* *} P<0.01,{ }^{* *} P<0.001$ denote significant differences between the groups.

\section{ABBREVIATIONS}

AIC, AICAR; AMPK, AMP-activated protein kinase; DN, dominant negative; DKO, double knockout; DON, 6-diazo-5-oxo-L-norleucine; EV, empty vector; EGCG, epigallocatechin 3-gallate; Glu, glucose; Gln, glutamine; Lact, lactate; $\mathrm{MEF}$, mouse embryonic fibroblasts; MFI, mean fluorescence intensity; MMP, mitochondrial membrane potential; mTOR, mammalian target of rapamycin; Oli, oligomycin; OXPHOS, oxidative phosphorylation; Palm, palmitate; PGC-1a, peroxisome proliferator-activated receptor coactivator-1; Rap, rapamycin; S.D., standard deviation; WT, wild type.

\section{ACKNOWLEDGEMENTS}

We thank Dr. SC Mande, Director, NCCS, Pune, India, and Dr. GC Mishra, former Director, NCCS, Pune, India for being very supportive and giving all the encouragement to carry out this work. We also thank Dr. David Carling (Imperial College School of Medicine, London, UK) for providing Myc-tagged dominantnegative-AMPK (AMPK-DN) and constitutively active-AMPK constructs. BC and NM thank the Council of Scientific and Industrial Research (CSIR) India; PM and SVS thank the University Grant Commission (UGC), India for providing fellowship. The support from the other lab members and colleagues at NCCS as well as staff members from the Experimental Animal Facility, FACS, Confocal and mass spectrometry facility is duly acknowledged. This work was supported by intramural grant from NCCS funded Department of Biotechnology, Government of India. This work was carried out as a part of fulfillment of the Ph.D. thesis of BC to be submitted to the Savitribai Phule Pune University, Pune, India.

\section{COMPETING INTERESTS}

The authors declare no conflict of interest.

\section{REFERENCES}

1 Vander Heiden MG, Cantley LC, Thompson CB. Understanding the Warburg effect: the metabolic requirements of cell proliferation. Science 2009; 324: 1029-1033.

2 Warburg O. On the origin of cancer cells. Science 1956; 123: 309-314.

3 Koppenol WH, Bounds PL, Dang CV. Otto Warburg's contributions to current concepts of cancer metabolism. Nat Rev Cancer 2011; 11: 325-337.

4 LeBleu VS, O'Connell JT, Gonzalez Herrera KN, Wikman H, Pantel K, Haigis MC et al. PGC-1alpha mediates mitochondrial biogenesis and oxidative phosphorylation in cancer cells to promote metastasis. Nat Cell Biol 2014; 16: 992-1003, 1001-1015.

5 Ward PS, Thompson CB. Metabolic reprogramming: a cancer hallmark even warburg did not anticipate. Cancer Cell 2012; 21: 297-308.

6 Weinberg F, Hamanaka R, Wheaton WW, Weinberg S, Joseph J, Lopez M et al. Mitochondrial metabolism and ROS generation are essential for Kras-mediated tumorigenicity. Proc Natl Acad Sci USA 2010; 107: 8788-8793.

7 Carmeliet P, Jain RK. Molecular mechanisms and clinical applications of angiogenesis. Nature 2011; 473: 298-307.

8 Bergeron R, Ren JM, Cadman KS, Moore IK, Perret P, Pypaert M et al. Chronic activation of AMP kinase results in NRF-1 activation and mitochondrial biogenesis. Am J Physiol Endocrinol Metab 2001; 281: E1340-E1346.

9 Stride N, Larsen S, Treebak JT, Hansen CN, Hey-Mogensen M, Speerschneider T et al. 5'-AMP activated protein kinase is involved in the regulation of myocardial beta-oxidative capacity in mice. Front Physiol 2012; 3: 33.

10 Williams RS, Salmons S, Newsholme EA, Kaufman RE, Mellor J. Regulation of nuclear and mitochondrial gene expression by contractile activity in skeletal muscle. J Biol Chem 1986; 261: 376-380.

11 Hardie DG. AMP-activated protein kinase: an energy sensor that regulates all aspects of cell function. Genes Dev 2011; 25: 1895-1908.

12 Hardie DG, Carling D. The AMP-activated protein kinase-fuel gauge of the mammalian cell? Eur J Biochem 1997; 246: 259-273.

13 Laplante M, Sabatini DM. mTOR signaling in growth control and disease. Cell 2012; 149: 274-293.

14 Kemp BE, Mitchelhill Kl, Stapleton D, Michell BJ, Chen ZP, Witters LA. Dealing with energy demand: the AMP-activated protein kinase. Trends Biochem Sci 1999; 24 22-25.
15 Salt IP, Johnson G, Ashcroft SJ, Hardie DG. AMP-activated protein kinase is activated by low glucose in cell lines derived from pancreatic beta cells, and may regulate insulin release. Biochem $J$ 1998; 335: 533-539.

16 Winder WW, Holmes BF, Rubink DS, Jensen EB, Chen M, Holloszy JO. Activation of AMP-activated protein kinase increases mitochondrial enzymes in skeletal muscle. J Appl Physiol 2000; 88: 2219-2226.

$17 \mathrm{Wu} \mathrm{H}$, Kanatous SB, Thurmond FA, Gallardo T, Isotani E, Bassel-Duby R et al. Regulation of mitochondrial biogenesis in skeletal muscle by CaMK. Science 2002; 296: 349-352.

$18 \mathrm{Wu}$ Z, Puigserver P, Andersson U, Zhang C, Adelmant G, Mootha V et al. Mechanisms controlling mitochondrial biogenesis and respiration through the thermogenic coactivator PGC-1. Cell 1999; 98: 115-124.

19 Choo AY, Kim SG, Vander Heiden MG, Mahoney SJ, Vu H, Yoon SO et al. Glucose addiction of TSC null cells is caused by failed mTORC1-dependent balancing of metabolic demand with supply. Mol Cell 2010; 38: 487-499.

20 Fillmore N, Jacobs DL, Mills DB, Winder WW, Hancock CR. Chronic AMP-activated protein kinase activation and a high-fat diet have an additive effect on mitochondria in rat skeletal muscle. J Appl Physiol 2010; 109: 511-520.

21 Garcia-Roves PM, Osler ME, Holmstrom MH, Zierath JR. Gain-of-function R225Q mutation in AMP-activated protein kinase gamma3 subunit increases mitochondrial biogenesis in glycolytic skeletal muscle. J Biol Chem 2008; 283: 35724-35734.

22 Zong $\mathrm{H}$, Ren JM, Young LH, Pypaert $M, M u J$, Birnbaum $M J$ et al. AMP kinase is required for mitochondrial biogenesis in skeletal muscle in response to chronic energy deprivation. Proc Natl Acad Sci USA 2002; 99: 15983-15987.

23 Barger PM, Browning AC, Garner AN, Kelly DP. p38 mitogen-activated protein kinase activates peroxisome proliferator-activated receptor alpha: a potential role in the cardiac metabolic stress response. J Biol Chem 2001; 276: 44495-44501.

24 Gibala MJ, McGee SL, Garnham AP, Howlett KF, Snow RJ, Hargreaves M. Brief intense interval exercise activates AMPK and p38 MAPK signaling and increases the expression of PGC-1alpha in human skeletal muscle. J Appl Physiol 2009; 106 929-934.

25 Jager S, Handschin C, St-Pierre J, Spiegelman BM. AMP-activated protein kinase (AMPK) action in skeletal muscle via direct phosphorylation of PGC-1alpha. Proc Natl Acad Sci USA 2007; 104: 12017-12022.

26 Lee WJ, Kim M, Park HS, Kim HS, Jeon MJ, Oh KS et al. AMPK activation increases fatty acid oxidation in skeletal muscle by activating PPARalpha and PGC-1. Biochem Biophys Res Commun 2006; 340: 291-295.

27 Suwa M, Egashira T, Nakano H, Sasaki H, Kumagai S. Metformin increases the PGC-1alpha protein and oxidative enzyme activities possibly via AMPK phosphorylation in skeletal muscle in vivo. J Appl Physiol 2006; 101: 1685-1692.

28 Marsin AS, Bertrand L, Rider MH, Deprez J, Beauloye C, Vincent MF et al. Phosphorylation and activation of heart PFK-2 by AMPK has a role in the stimulation of glycolysis during ischaemia. Curr Biol 2000; 10: 1247-1255.

29 Wu SB, Wei YH. AMPK-mediated increase of glycolysis as an adaptive response to oxidative stress in human cells: implication of the cell survival in mitochondrial diseases. Biochim Biophys Acta 2012; 1822: 233-247.

30 Kahn BB, Alquier T, Carling D, Hardie DG. AMP-activated protein kinase: ancient energy gauge provides clues to modern understanding of metabolism. Cell Metab 2005; 1: 15-25.

31 Jones RG, Plas DR, Kubek S, Buzzai M, Mu J, Xu Y et al. AMP-activated protein kinase induces a p53-dependent metabolic checkpoint. Mol Cell 2005; 18 283-293.

32 Laderoute KR, Amin K, Calaoagan JM, Knapp M, Le T, Orduna J et al. 5'-AMPactivated protein kinase (AMPK) is induced by low-oxygen and glucose deprivation conditions found in solid-tumor microenvironments. Mol Cell Biol 2006; 26: 5336-5347.

33 Jeon SM, Chandel NS, Hay N. AMPK regulates NADPH homeostasis to promote tumour cell survival during energy stress. Nature 2012; 485: 661-665.

34 Le A, Lane AN, Hamaker M, Bose S, Gouw A, Barbi J et al. Glucose-independent glutamine metabolism via TCA cycling for proliferation and survival in B cells. Cell Metab 2012; 15: 110-121.

35 Semenza GL. Tumor metabolism: cancer cells give and take lactate. J Clin Invest 2008; 118: 3835-3837.

36 Zaugg K, Yao Y, Reilly PT, Kannan K, Kiarash R, Mason J et al. Carnitine palmitoyltransferase $1 \mathrm{C}$ promotes cell survival and tumor growth under conditions of metabolic stress. Genes Dev 2011; 25: 1041-1051.

37 Jones AW, Yao Z, Vicencio JM, Karkucinska-Wieckowska A, Szabadkai G. PGC-1 family coactivators and cell fate: roles in cancer, neurodegeneration, cardiovascular disease and retrograde mitochondria-nucleus signalling. Mitochondrion 2012; 12: 86-99. 
38 Klimcakova E, Chenard V, McGuirk S, Germain D, Avizonis D, Muller WJ et al. PGC-1alpha promotes the growth of ErbB2/Neu-induced mammary tumors by regulating nutrient supply. Cancer Res 2012; 72: 1538-1546.

39 McGuirk S, Gravel SP, Deblois G, Papadopoli DJ, Faubert B, Wegner A et al. PGC-1a supports glutamine metabolism in breast cancer. Cancer Metab 2013; 1: 22.

40 Lerner C, Bitto A, Pulliam D, Nacarelli T, Konigsberg M, Van Remmen $\mathrm{H}$ et al. Reduced mammalian target of rapamycin activity facilitates mitochondrial retrograde signaling and increases life span in normal human fibroblasts. Aging Cell 2013; 12: 966-977.

41 Li J, Miller EJ, Ninomiya-Tsuji J, Russell RR 3rd, Young LH. AMP-activated protein kinase activates $\mathrm{p} 38$ mitogen-activated protein kinase by increasing recruitment of p38 MAPK to TAB1 in the ischemic heart. Circ Res 2005; 97: 872-879.

42 Chuang HC, Chou CC, Kulp SK, Chen CS. AMPK as a potential anticancer targetfriend or foe? Curr Pharm Des 2014; 20: 2607-2618.

43 Hardie DG. The LKB1-AMPK pathway-friend or foe in cancer? Cancer Cell 2013; 23: 131-132.

44 Wada T, Penninger JM. Mitogen-activated protein kinases in apoptosis regulation. Oncogene 2004; 23: 2838-2849.

45 Porras A, Zuluaga S, Black E, Valladares A, Alvarez AM, Ambrosino C et al. P38 alpha mitogen-activated protein kinase sensitizes cells to apoptosis induced by different stimuli. Mol Biol Cell 2004; 15: 922-933.
46 Juretic N, Santibanez JF, Hurtado C, Martinez J. ERK 1,2 and p38 pathways are involved in the proliferative stimuli mediated by urokinase in osteoblastic SaOS-2 cell line. J Cell Biochem 2001; 83: 92-98.

47 Liu B, Fang M, Lu Y, Mills GB, Fan Z. Involvement of JNK-mediated pathway in EGF-mediated protection against paclitaxel-induced apoptosis in SiHa human cervical cancer cells. Br J Cancer 2001; 85: 303-311.

48 Malvi P, Chaube B, Pandey V, Vijayakumar MV, Boreddy PR, Mohammad N et al. Obesity induced rapid melanoma progression is reversed by orlistat treatment and dietary intervention: role of adipokines. Mol Oncol 2015; 9: 689-703.

49 Spinazzi M, Casarin A, Pertegato V, Salviati L, Angelini C. Assessment of mitochondrial respiratory chain enzymatic activities on tissues and cultured cells. Nat Protoc 2012; 7: 1235-1246.

This work is licensed under a Creative Commons Attribution 4.0 International License. The images or other third party material in this article are included in the article's Creative Commons license, unless indicated otherwise in the credit line; if the material is not included under the Creative Commons license, users will need to obtain permission from the license holder to reproduce the material. To view a copy of this license, visit http://creativecommons.org/licenses/ by/4.0/

Supplementary Information accompanies the paper on the Cell Death and Discovery website (http://www.nature.com/cddiscovery) 